\title{
Quantum Isomorphic Shell Model: Multi-Harmonic Shell Clustering of Nuclei
}

\author{
G. S. Anagnostatos \\ Institute of Nuclear Physics, National Center for Scientific Research Demokritos, Greece \\ Email: anagnos4@otenet.gr
}

Received 2013

\begin{abstract}
The present multi-harmonic shell clustering of a nucleus is a direct consequence of the fermionic nature of nucleons and their average sizes. The most probable form and the average size for each proton or neutron shell are here presented by a specific equilibrium polyhedron of definite size. All such polyhedral shells are closely packed leading to a shell clustering of a nucleus. A harmonic oscillator potential is employed for each shell. All magic and semi-magic numbers, g.s. single particle and total binding energies, proton, neutron and mass radii of ${ }^{40} \mathrm{Ca},{ }^{48} \mathrm{Ca},{ }^{54} \mathrm{Fe},{ }^{90} \mathrm{Zr},{ }^{108} \mathrm{Sn},{ }^{114} \mathrm{Te},{ }^{142} \mathrm{Nd}$, and ${ }^{208} \mathrm{~Pb}$ are very successfully predicted.
\end{abstract}

Keywords: Cluster Models; ${ }^{40} \mathrm{Ca},{ }^{48} \mathrm{Ca},{ }^{54} \mathrm{Fe},{ }^{90} \mathrm{Zr},{ }^{108} \mathrm{Sn},{ }^{114} \mathrm{Te},{ }^{142} \mathrm{Nd},{ }^{208} \mathrm{~Pb}$, Binding Energies; Coulomb Energies; Proton; Neutron; Mass Radii; Atomic Fermions

\section{Introduction}

The present work supports a shell clustering of nuclear structure by taking advantage mainly of two fundamental properties of nucleons. The first is the fermionic nature of nucleons and the second is that nucleons possess finite average sizes. As will be explained shortly [see a) and b) below], the first leads to the shell structure of the nucleus and the second to the average nuclear sizes. These properties taken together lead to the aforementioned shell clustering of the nucleus. Indeed:

a) The fermionic nature of nucleons, due to their antisymmetric wave function, makes them behave as if a repulsive force (of unknown nature) is acting among them. [1]. This repulsive property of nucleons is derived not only by this antisymmetrization, but also by the repulsive character of the nuclear force itself, considered as a result of the quark structure of the nucleons. The existence of this force shifts the nuclear many-body problem to the problem of finding the equilibria of repulsive particles on a sphere, like the sphere of a nuclear shell. According to [2], such equilibria are possible only for specific numbers of particles and when these particles have most probable positions at the vertices, or middles of faces, or middles of edges, or simultaneously at these characteristic points of regular polyhedra or their derivative polyhedra. Such polyhedra in the present model stand for the most probable forms of nuclear shells which - taken in specific sequence, as we will see below - precisely reproduce the nuclear magic numbers, with no use of the strong spin-orbit coupling. It is essential to emphasize here that the structures of these polyhedra precisely possess the quantization of orbital angular momentum [3].

b) In addition, the finite average size of nucleons, together with the above most probable polyhedral structure of shells, leads to the average sizes of all nuclear shells and thus of all nuclei. This is obtained by packing the shells themselves, i.e., each polyhedral shell reaches its minimum size, that is, the bags of nucleons at its vertices come in contact with the bags of nucleons at the vertices of a previous polyhedron.

However, it should be mentioned that the foundation of the shell structure of the nucleus is offered by other theories as well, where the antisymmetrization of the nucleon wave function and the nuclear interaction play an important role.

The present approach, where the nuclear structure is qualitatively derived without reference to the inter-particle forces, is applicable not only in nuclear physics but also in any other branch of physics where fermions of definite average size are the constituent particles. Take, for example, cluster physics when the constituent particles are atoms with half-integer spins; thus they could be considered as atomic fermions. Thus, the present paper can guide research in other fields, as well as in nuclear physics.

\section{The Model and Applications}




\subsection{Specification of Most Probable Forms and Average Sizes of Nuclear Shells}

As mentioned in the introduction the fermionic nature of nucleons and their average sizes lead to the polyhedral average shell structure of a nucleus. This structure is different for neutrons and protons, since these two kinds of nucleons are considered as two different sets of particles. Indeed, this problem for one set or for two different sets of repulsive particles on a sphere or on concentric spheres (like the shapes of closed nuclear shells) was solved by Leech long ago [2]. According to this reference, each of these two sets should be in equilibrium by itself on a sphere or on concentric spheres and the resulting two structures should be in a new relative equilibrium as well.

As aforementioned in the introduction, these equilibria are obtained at the vertices or middles of faces or middles of edges of regular polyhedra and their derivative polyhedra. However, when we consider the middles of faces or the middles of edges of any of the aforementioned polyhedra, we obtain the vertices of another regular or semi-regular polyhedron. Thus, we see that it is sufficient to consider only the vertices of polyhedra. These polyhedra are the zerohedron, the octahedron, the hexahedron (cube), the cuboctahedron, the icosahedron, the dodecahedron, the rhombic cuboctahedron, the icosidodecahedron, and the rhombic triacondahedron with numbers of vertices $2,6,8,12,12,20,24,30$, and 32 , respectively. These polyhedra can appear more than one time. Among them three polyhedra can be analysed as two symmetric, equilibrium structures with smaller numbers of vertices having together the same central, angular structure as the initial polyhedron. Namely, the dodecahedron (20 vertices) can be analysed as a cube ( 8 vertices) and the remaining (12 vertices), the icosidodecahedron (30 vertices) can be analysed as an octahedron (6 vertices) and the remaining (24 vertices), and the rhombic triacondahedron (32 vertices) can be analysed as an icosahedron (12 vertices) and a dodecahedron (20 vertices) which can further be analysed as above. Recommended books for a sufficient familiarity with regular and semiregular polyhedra are $[4,5]$, the former being simple and very instructive and the latter somewhat more sophisticated.

Since both the initial equilibrium polyhedra and the polyhedra derived by their aforementioned analyses are candidates to present the most probable forms of nuclear shells and sub-shells, what is necessary in the following is to describe how these polyhedra are concentrically and most symmetrically [6] arranged in space to present nuclear shells and sub-shells.

First, we start with the polyhedra with smaller numbers of vertices, i.e., with 2, 6, 8, 12, and 20 vertices. Among them we assign the polyhedra with triangular faces (corresponding to stable equilibria [2] and having number of vertices 2,6 , and 12), i.e., the zerohedron, the octahedron, and the icosahedron, to the first three neutron shells and the remaining polyhedra (corresponding to unstable equilibria [2] and having number of vertices 2 , 8 , and 20), i.e., the zerohedron, the hexahedron (cube), and the dodecahedron, to the first three proton shells (see Figure 1). This assignment makes each neutron shell have a corresponding proton shell with the same rotational symmetry, i.e., a neutron zerohedron corresponds to a proton zerohedron, a neutron octahedron to a proton hexahedron, and a neutron icosahedron to a proton dodecahedron. This assignment, in addition, minimizes the Coulomb energy, since the aforementioned polyhedra for protons are of a larger size than those assigned for neutrons (see Figure 1). This type of correspondence between neutron and proton shells is valid for the whole periodic table of nuclei.

Then, their average sizes are determined with respect to the average size of a proton $\left(r_{p}=0.860 \mathrm{fm}\right)$ and that of a neutron $\left(r_{n}=0.974 \mathrm{fm}\right)$, taken as the only universal size parameters of the model. This happens by packing the candidate shells themselves and following the instructtions of the next section for all candidate shells in Figure 1

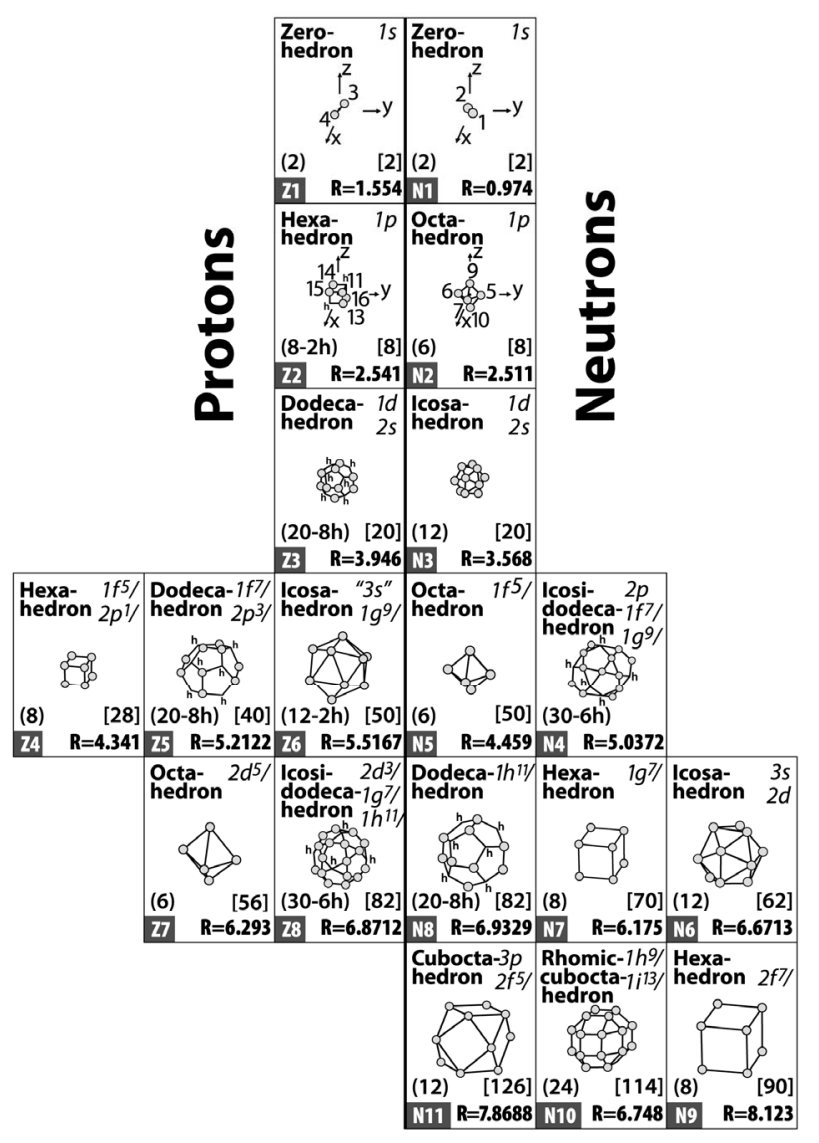

Figure 1. Most probable forms and average sizes of nuclear shells and sub-shells for nuclei up to $Z=82$ and $N=126$. 
1. In Table 1 the average shells of Figure 1 which are in contact with each other are given. Specifically, this table provides what polyhedron is in contact with a previous one. In general, the prefix $\mathrm{Z}$ stands for a proton polyhedron and the prefix $\mathrm{N}$ for a neutron polyhedron.

In Figure 1 the dodecahedra, the icosidodecahedra, and the rhombic triacondahedra are always analysed as mentioned earlier. These analyses lead to the maximum compactness. All octahedra and all cubes in the figure have parallel edges. However, the dodecahedron Z3 has parallel edges only with the dodecahedron N8 but not with dodecahedron Z5. The orientation of Z5 is derived by rotating either $\mathrm{Z} 3$ or $\mathrm{N} 890^{\circ}$ around the $\mathrm{z}$ axis. Similarly, the icosahedron N3 has parallel edges only with the icosahedron N6 but not with icosahedron Z6. The orienttation of Z6 is also derived by rotating N3 or N6 $90^{\circ}$ around the $\mathrm{z}$ axis. Also, the orientation of icosidodecahedron $\mathrm{Z} 8$ is derived from that of icosidodecahedron N4 by considering similar rotation i.e., again $90^{\circ}$. These rotations lead to pairs of similar polyhedra with perpendicular edges. All these rotations favour the maximum compactness of the polyhedra involved.

It is worth mentioning that the three proton polyhedra $\mathrm{Z4}, \mathrm{Z5}$, and Z6 are the results of analysis of a rhombic triacondahedron maintaining the same central, angular structure, as the three neutron polyhedra N6, N7, and N8 are. Each of these two rhombic triacondahedra could be derived by rotating the one with respect to the other by $90^{\circ}$ around the $\mathrm{z}$ axis. Similarly, the polyhedra N4 and $\mathrm{N} 5$, and the polyhedra $\mathrm{Z7}$ and $\mathrm{Z8}$ are the results of analysis of two icosidodecahedra with orientations differing by $90^{\circ}$ as well. Finally, the polyhedra N9, N10 and N11 are angularly related polyhedra. Indeed, the middles of edges of the cube N9 form the cuboctahedron N11 and the middles of faces of $\mathrm{N} 11$ form the rhombic cuboctahedron N10. In conclusion, each row of neutron or proton poly- hedra in Figure 1 angularly refers to one and only one polyhedron which is analysed as shown in the figure. As mentioned earlier, each row in Figure 1 of neutron (proton) polyhedra corresponds to an opposite row of proton (neutron) polyhedra which have the same rotational symmetry. In geometrical language, polyhedra with the same rotational symmetry are called reciprocal.

As we can see in Figure 1, the numbers inside brackets in the block of a polyhedron close to the central vertical line of the figure, which are equal to the sum of vertices of all previous polyhedra including this polyhedron, coincide with the magic numbers for protons and neutrons, i.e., $2,8,20,50,82$, and 126 . The numbers inside brackets in the remaining blocks of the figure stand for the semi-magic numbers 28 and 40 for protons, and 62, 70, 90, and 114 for neutrons. The numbers in the blocks of $\mathrm{N} 4$ and Z7 do not form semi-magic numbers since the corresponding polyhedra are not present in all cases. As will be understood later, in many cases the octahedra N4 and $\mathrm{Z7}$ remain as part of the structure of the neighbouring icosidodecahedra $\mathrm{N} 5$ and Z8, respectively. The places of their vertices in the neighbouring polyhedron are marked with $\mathrm{h}$ (hole) and form a similar polyhedron, as happens in all other cases of analyses of polyhedra mentioned earlier.

The successful reproduction of magic and semi-magic numbers by the polyhedra of Figure 1 permits a tentative assignment of quantum states to the polyhedra vertices standing as average positions of nucleons. One rule is that quantum sub-shells are always assigned at the vertices of the same polyhedron. A test of this tentative assignment of quantum states to the vertices of polyhedra will be made in the section of the quantum mechanical treatment of the model below. In the future, this assignment will be further and further tested by employing more and more nuclear observables.

Table 1. Initial and final radii in Fermi of polyhedra whose bags at their vertices are in contact.

\begin{tabular}{cccccccc}
\hline \multicolumn{2}{c}{ Initial } & \multicolumn{2}{c}{ Final } & \multicolumn{2}{c}{ Final } & \multicolumn{2}{c}{ Final } \\
\hline Polyh. & Radius & Polyh. & Radius & Polyh. & Radius & Polyh. & Radius \\
N1 & 0.974 & Z1 & 1.554 & Z2 & 2.541 & N2 & 2,511 \\
N2 & 2.511 & Z3 & 3.946 & N3 & 3.568 & N5 & 4.459 \\
Z2 & 2.541 & Z4 & 4.341 & & & & \\
Z3 & 3.946 & Z6 & 5.5167 & & & & \\
N3 & 3.568 & Z5 & 5.2122 & & & & \\
Z4 & 4.341 & N4 & 5.0372 & N7 & 6.175 & & \\
N5 & 4.459 & Z7 & 6.293 & & & & \\
N4 & 5.0372 & Z8 & 6.8712 & N10 & 6.748 & & \\
Z5 & 5.2122 & N6 & 6.6713 & & & & \\
Z6 & 5.5167 & N8 & 6.9329 & & & & \\
N7 & 6.175 & N9 & 8.123 & & & & \\
N8 & 6.9329 & N11 & 7.8688 & & & & \\
\hline
\end{tabular}


In Figure 1 the high-symmetry equilibrium polyhedra [2] employed by the model to represent the most probable forms and average sizes of all nuclear shells and sub-shells up to $Z=82$ and $N=126$ are given. Specifically, at the top of each block of the figure the name of the polyhedron shown in this block (left) and the quantum states of the nucleons accommodated by this polyhedron (right) are listed, separately for protons and neutrons. In addition at the bottom-left of each block, in a black square, the numbering of this polyhedron in successive order of filling, preceded with the letter $\mathrm{Z}$ for protons and with the letter $\mathrm{N}$ for neutrons, is given. Over this black square the number of the polyhedral vertices, and the number of the possible unoccupied vertices characterized as holes, h (symmetrically distributed and forming an equilibrium polyhedron themselves), are also given in parentheses. At the bottom-right of each block the radius of the polyhedron in units Fermi is listed. Over this radius the cumulative number of vertices of all previous polyhedra and this polyhedron is also given in brackets.

The pieces of information given in this section are sufficient, if someone wants to redefine the most probable forms and the average sizes of equilibrium polyhedra of Figure 1 employed as the average forms of nuclear shells. However, if someone just wants to apply the model to any nuclear observable, it is sufficient to take the polyhedra of this figure for granted and not to get involved with its derivation.

\subsection{Radii of Polyhedra and Coordinates of Their Vertices}

Figure 2 is employed to facilitate the present section. The sphere labelled 1 (with coordinates of its center $\mathrm{x}_{0}$, $\mathrm{y}_{0}, \mathrm{z}_{0}$ ) stands for an average nucleon position at a vertex of a polyhedron with known radius $\mathrm{R}_{0}$. The sphere labelled 2 (considered in contact with the previous sphere and with coordinates of each center $\mathrm{x}, \mathrm{y}, \mathrm{z}$ ) stands for an average nucleon position at a vertex of another polyhedron with unknown radius $\mathrm{R}_{\mathrm{x}}$.

Apparently, among the above totally six coordinates and the distance $d_{12}$ between the centers of the two spheres 1 and 2, Eq. (1) is valid

$$
\left(\mathrm{x}-\mathrm{x}_{0}\right)^{2}+\left(\mathrm{y}-\mathrm{y}_{0}\right)^{2}+\left(\mathrm{z}-\mathrm{z}_{0}\right)^{2}=\mathrm{d}_{12}{ }^{2},
$$

On the line $\mathrm{O} 1$ we assume the vertex called 1 (with coordinates $\mathrm{x}_{1}, \mathrm{y}_{1}, \mathrm{z}_{1}$ ) of a polyhedron which has the symmetries of the polyhedron with known radius $\mathrm{R}_{0}$. On the line $\mathrm{O} 2$ we assume the vertex called 2 (with coordinates $\mathrm{x}_{2}, \mathrm{y}_{2}, \mathrm{z}_{2}$ ) of a polyhedron which has the symmetries of the polyhedron with unknown radius $\mathrm{R}_{\mathrm{x}}$. It is apparent that the coordinates $\mathrm{x}_{0}, \mathrm{y}_{0}, \mathrm{z}_{0}$ and $\mathrm{x}, \mathrm{y}, \mathrm{z}$ can be expressed with respect to the coordinates $\mathrm{x}_{1}, \mathrm{y}_{1}, \mathrm{z}_{1}$ and $\mathrm{x}_{2}$, $\mathrm{y}_{2}, \mathrm{z}_{2}$, and the radii $\mathrm{R}_{0}$ and $\mathrm{R}_{\mathrm{x}}$ as follows:

$$
\begin{aligned}
& \mathrm{x}_{0}=\mathrm{R}_{0} * \mathrm{x}_{1} / \mathrm{R}_{1}, \mathrm{y}_{0}=\mathrm{R}_{0} * \mathrm{y}_{1} / \mathrm{R}_{1}, \mathrm{z}_{0}=\mathrm{R}_{0} * \mathrm{z}_{1 /} \mathrm{R}_{1} \\
& \mathrm{x}=\mathrm{R}_{\mathrm{x}}^{*} \mathrm{x}_{2} / \mathrm{R}_{2}, \mathrm{y}=\mathrm{R}_{\mathrm{x}}^{*} \mathrm{y}_{2} / \mathrm{R}_{2}, \mathrm{z}=\mathrm{R}_{\mathrm{x}}^{*} \mathrm{z}_{2} / \mathrm{R}_{2} \\
& \mathrm{R}_{0}{ }^{2}=\mathrm{x}_{0}{ }^{2}+\mathrm{y}_{0}{ }^{2}+\mathrm{z}_{0}{ }^{2}, \mathrm{R}_{\mathrm{x}}{ }^{2}=\mathrm{x}^{2}+\mathrm{y}^{2}+\mathrm{z}^{2}, \\
& \mathrm{R}_{1}{ }^{2}=\mathrm{x}_{1}{ }^{2}+\mathrm{y}_{1}{ }^{2}+\mathrm{z}_{1}{ }^{2}, \mathrm{R}_{2}{ }^{2}=\mathrm{x}_{2}{ }^{2}+\mathrm{y}_{2}{ }^{2}+\mathrm{z}_{2}{ }^{2}
\end{aligned}
$$

By substituting the above relationships to Eq.(1) finally we obtain Eq.(3)

$$
\begin{aligned}
& \mathrm{R}_{x}^{2}-\left[\left(\mathrm{x}_{1} \mathrm{x}_{2}+\mathrm{y}_{1} \mathrm{y}_{2}+\mathrm{z}_{1} \mathrm{z}_{2}\right) * 2 \mathrm{R}_{0} /\left(\mathrm{R}_{1} \mathrm{R}_{2}\right)\right] \mathrm{R}_{\mathrm{x}} \\
& +\left[\mathrm{R}_{0}^{2}-\mathrm{d}_{12}^{2}\right]=0
\end{aligned}
$$

The quantity $\mathrm{d}_{12}$ takes on the following numerical values:

$d_{12}=r_{n}+r_{n}=1.948 f m$ when two neutron bags are in contact,

$\mathrm{d}_{12}=\mathrm{r}_{\mathrm{n}}+\mathrm{r}_{\mathrm{p}}=1.834 \mathrm{fm}$ when a neutron bag is in contact with a proton bag, and

$\mathrm{d}_{12}=2^{*}<\mathrm{r}^{2}>^{\mathrm{ch}}=1.800 \mathrm{fm}$ when two proton bags are in contact,

where $<\mathrm{r} 2>^{\text {ch }}=0.900 \mathrm{fm}$ is the average proton charge radius from the literature [7].

By employing Eq.(3) we can determine the radius of the new polyhedron $R_{x}$ with respect to the radius of the known polyhedron $\mathrm{R}_{0}$ and the coordinates $\mathrm{x}_{1}, \mathrm{y}_{1}, \mathrm{z}_{1}$ and $\mathrm{x}_{2}, \mathrm{y}_{2}, \mathrm{z}_{2}$ of the two auxiliary polyhedra employed. These coordinates for all kinds of polyhedra involved in Figure $\mathbf{1}$ are given in Table $\mathbf{2}$ together with corresponding radii $R_{1}$ and $R_{2}$ necessary for the application of Eq.(3).

If Eq. (3) does not have a solution, then there is no contact between bag 1 of the known polyhedron and bag 2 chosen at a specific vertex of the candidate polyhedron. Working in same way, we can check whether there is a contact of bag 1 with bag 2 considered at another vertex of the same candidate polyhedron. Usually, we can foresee which vertex of the polyhedron under investigation is closer to the chosen vertex of the known polyhedron and thus one trial is enough. In general, a simple computer program can easily solve the problem employing all vertices of the candidate polyhedron simultaneously.

As mentioned, the sequences of polyhedra whose bags at their vertices are in contact with those of previous polyhedra are given in Table 1. In this table each polyhedron used as known polyhedron with radius $\mathrm{R}_{0}$ (called initial polyhedron) and the derived polyhedron with radius $R_{x}$ (called final polyhedron) are shown together with their radii. The radius $R_{x}$ is derived by taking the polyhedron of the same row in the table as initial one and applying Eqs.(1-3). As shown in the table there are cases where one specific polyhedron used as an initial polyhedron leads to the derivation of two or three final polyhedra of different symmetry. The described procedure strictly leads to the maximum possible density of polyhedra standing as the most probable forms and average sizes of shells and sub-shells for fermions. No overlap- 
ping of bags appears among any bags at the vertices of the polyhedra in Figure 1. In general, the polyhedral average shell structure presented in Figure 1 is unique.

The coordinates of all average positions for nucleons in closed shells for nuclei up $\mathrm{Z}=82$ and $\mathrm{N}=126$ are obtained by considering the proper coordinates from Table 2 and then multiplying by the radius of the polyhedron of interest from Figure 1.

\subsection{Multi-harmonic Treatment of a Nucleus}

The analytical part of the model assumes a harmonic oscillator potential for the nucleons of each proton or neutron shell (and not for all nucleons in a nucleus):

$$
\mathrm{V}(\mathrm{r})=\mathrm{v}_{0}+1 / 2 \mathrm{m \omega ^{2 }} \mathrm{r}^{2},
$$

where $\mathrm{v}_{0}$ and $\omega$ are different parameters for each proton or neutron shell. That is, the potential form is the same for all nucleons, but its parameters are different for each proton or neutron shell. Due to the two assumptions below, however, the final number of parameters is substantially reduced.

a) The $\hbar \omega$, for each shell, is determined [8] according to Eq.(5)

$$
\hbar \omega=\left(\hbar^{2} / m\right)(n+3 / 2) /<r^{2}>,
$$

where $\left.<\mathrm{r}^{2}\right\rangle^{1 / 2}$ is the average size of a specific proton or neutron shell which remains constant for all nuclei. All these sizes are given in Figure 1. The only parameters necessary in determining these sizes are the two size parameters $r_{p}$ and $r_{n}$, as discussed in section 2.2.

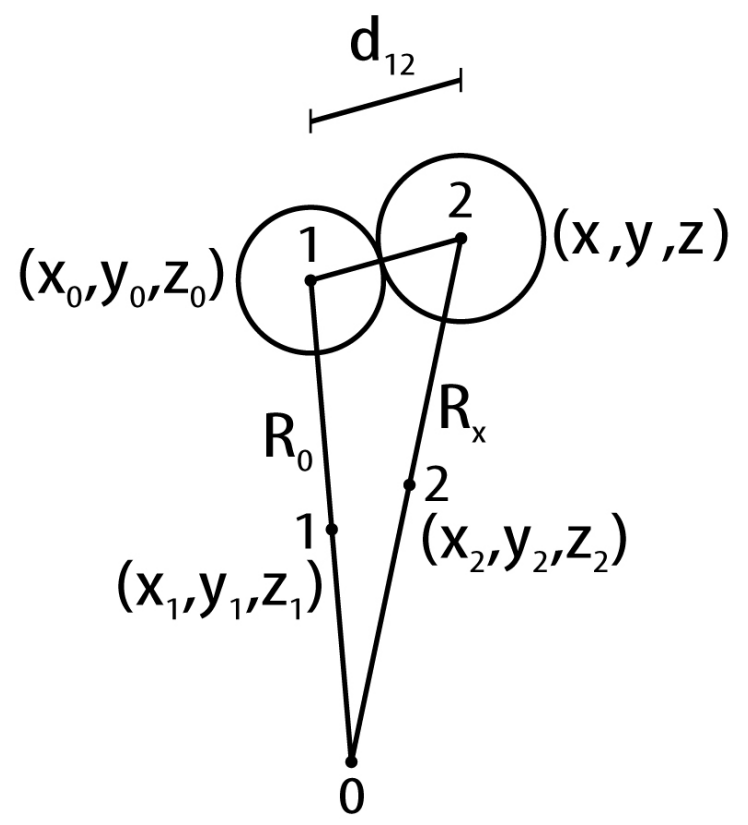

Figure 2. Determination of the radius of a polyhedron with respect to the radius of another polyhedron when nucleon bags at their vertices are in contact.

Table 2. Standard coordinates and corresponding radii of all polyhedra employed by the model for nuclei up $\mathrm{Z}=82$ and $\mathrm{N}=$

\begin{tabular}{|c|c|c|c|}
\hline \multicolumn{2}{|c|}{ Polyhedron } & Standard coordinates & Radii \\
\hline \multicolumn{2}{|l|}{ Zerohedron $\mathrm{Z1}$} & $(1,-1,-1),(-1,1,1)$ & $\sqrt{3}$ \\
\hline \multicolumn{2}{|l|}{ N1 } & $(1,1,0),(-1,-1,0)$ & $\sqrt{2}$ \\
\hline \multicolumn{2}{|c|}{ Cube $\quad \mathrm{Z} 2, \mathrm{Z} 4, \mathrm{~N} 7, \mathrm{~N} 9$} & $( \pm 1, \pm 1, \pm 1)$ & $\sqrt{3}$ \\
\hline \multicolumn{2}{|c|}{ Octahedron $\mathrm{N} 2, \mathrm{~N} 5, \mathrm{Z7}$} & $( \pm 1,0,0),(0, \pm 1,0),(0,0, \pm 1)$ & 1 \\
\hline \multicolumn{2}{|c|}{ Dodecahedron Z3, N8 } & $\left( \pm \tau^{2}, \pm 1,0\right),\left( \pm 1,0, \pm \tau^{2}\right),(0, \pm \tau 2, \pm 1),( \pm \tau, \pm \tau, \pm \tau)$ & $\tau \sqrt{3}$ \\
\hline \multicolumn{2}{|c|}{$\mathrm{Z} 5$} & $\left(0, \pm 1, \pm \tau^{2}\right),\left(\left( \pm 1, \pm \tau^{2}, 0\right),(( \pm \tau 2,0, \pm 1),( \pm \tau, \pm \tau, \pm \tau)\right.$ & $\tau \sqrt{3}$ \\
\hline \multirow[t]{2}{*}{ Icosahedron } & N6 & $( \pm \tau, 0, \pm 1),(0, \pm 1, \pm \tau),( \pm 1, \pm \tau, 0)$ & $\sqrt{\tau+2}$ \\
\hline & & $(0, \pm \tau, \pm 1),( \pm \tau, \pm 1,0),( \pm 1,0, \pm \tau)$ & $\sqrt{\tau+2}$ \\
\hline \multirow[t]{2}{*}{ Icosidodecahedron } & N4 & $\begin{array}{c}\left( \pm 1, \pm \tau, \pm \tau^{2}\right),\left( \pm \tau, \pm \tau^{2}, \pm 1\right),( \pm \tau 2, \pm 1, \pm \tau) \\
\quad(\mathbf{0}, \mathbf{0}, \pm \mathbf{2} \tau),(\mathbf{0}, \pm \mathbf{2} \tau, \mathbf{0}),( \pm \mathbf{2} \tau, \mathbf{0}, \mathbf{0})\end{array}$ & $2 \tau$ \\
\hline & Z8 & $\begin{array}{c}\left( \pm \tau, \pm 1, \pm \tau^{2}\right),\left( \pm 1, \pm \tau^{2}, \pm \tau\right),\left( \pm \tau^{2}, \pm \tau, \pm 1\right) \\
\quad(\mathbf{0}, \mathbf{0}, \pm \mathbf{2} \tau),(\mathbf{0}, \pm \mathbf{2} \tau, \mathbf{0}),( \pm \mathbf{2} \tau, \mathbf{0}, \mathbf{0})\end{array}$ & $2 \tau$ \\
\hline Cuboctahedron & N11 & $( \pm 1,0, \pm 1),(0, \pm 1, \pm 1),( \pm 1, \pm 1,0)$ & $\sqrt{2}$ \\
\hline $\begin{array}{l}\text { Rhombic } \\
\text { Cuboctahedron }\end{array}$ & $\begin{array}{l}\text { N10 } \\
\text { N9 }\end{array}$ & {$[ \pm 1, \pm(\sqrt{2}-1), \pm(\sqrt{2}-1)],,[ \pm(\sqrt{2}-1), \pm(\sqrt{2}-1), \pm 1],[ \pm(\sqrt{2}-1), \pm 1, \pm(\sqrt{2}-1)]$} & $\sqrt{7-4 \sqrt{2}}$ \\
\hline
\end{tabular}
126 (see Figure 1). Symbol $\tau=(\sqrt{5}+1) / 2$. 
b) The parameter of the depth of the potential for each proton or neutron shell is determined according to Eq.(6)

$$
\begin{aligned}
E_{j} & =v_{j}-\hbar \omega_{j}\left(n_{j}+3 / 2\right)=v_{i}-\hbar \omega_{1}\left(n_{i}+3 / 2\right)=E_{i}, \text { or } \\
v_{j} & =v_{i}-\hbar \omega_{i}\left(n_{i}+3 / 2\right)+\hbar \omega_{j}\left(n_{j}+3 / 2\right)=v_{0}+\hbar \omega_{j}\left(n_{j}+3 / 2\right) .
\end{aligned}
$$

This assumption implies that all nucleons in a nucleus are equally bound in their own potentials (excluding Coulomb and spin-orbit interactions).

It is apparent from Eq.(6) that, since according to the previous assumption a) all $\hbar \omega$ are already determined as above by applying Eq.(5) with respect only of the two size parameters $r_{p}$ and $r_{n}$, the depth $v_{i}$ of the potential for each shell $i$ can be defined with the knowledge of only one additional parameter $\mathrm{V}_{0}$ (This is the third universal parameter of the present model equal to $40.268 \mathrm{MeV}$ ).

Solving Schroedinger's equation for the potential of Eq.(4), we obtain [8] the following equation

$$
\begin{aligned}
\mathrm{L}_{\mathrm{k}}^{\ell+1 / 2}(\mathrm{z})= & \left\{[\Gamma(1 / 2 \mathrm{~N}+1 / 2 \ell+1)]^{2} / \mathrm{k} ! \Gamma(\ell+3 / 2)\right\} \\
& { }_{1} \mathrm{~F}_{1}(-\mathrm{k} ; \ell+3 / 2 ; \mathrm{z}),
\end{aligned}
$$

where ${ }_{1} \mathrm{~F}_{1}(\alpha ; \mathrm{c} ; \mathrm{z})=1+(\alpha / \mathrm{c}) \mathrm{z}+\alpha(\alpha+1) \mathrm{z}^{2} / \mathrm{c}(\mathrm{c}+1) 2$ ! + $\alpha(\alpha+1)(\alpha+2) z^{3} / \mathrm{c}(\mathrm{c}+1)(\mathrm{c}+2) 3 !+\ldots$, with $\mathrm{c}=\ell+3 / 2, \alpha=$ $-\mathrm{k}, \mathrm{z}=2 \alpha \mathrm{r}^{2}$, and $\alpha=\mathrm{m} \omega / 2 \hbar$. The series terminates with the term $(-1)^{\mathrm{k}}[\Gamma(\mathrm{c}) / \Gamma(\mathrm{c}+\mathrm{k})] \mathrm{z}^{\mathrm{k}}$ and the various quantum numbers involved are $\mathrm{n}=0,1,2, \ldots ; \mathrm{k}=0,1,2, \ldots ; \mathrm{k} \leq \mathrm{n} / 2$, $\ell=0,1,2, \ldots, \ell \leq \mathrm{n}$, and $\ell=\mathrm{n}-2 \mathrm{k}[8]$.

The explicit forms of the first few equations of the wave functions for a harmonic oscillator potential derived from Eq.(7) can be found in several books of Quantum Mechanics and Nuclear Physics [8]. However, it is considered instructive for a complete list of all wave functions for nuclei up to $Z=126$ and $N=184$, derived from the recursion formula Eq.(7), to be shown below.

$$
\begin{aligned}
\mathrm{R}_{1 \mathrm{~s}}= & (128 / \pi)^{1 / 4} \alpha^{3 / 4} e^{-a r^{2}} \\
\mathrm{R}_{2 \mathrm{~s}}= & (128 / \pi)^{1 / 4}(3 / 2)^{1 / 2} \alpha^{3 / 4}\left(1-4 \alpha \mathrm{r}^{2} / 3\right) e^{-a r^{2}} \\
\mathrm{R}_{3 \mathrm{~s}}= & (128 / \pi)^{1 / 4}(15 / 8)^{1 / 2} \alpha^{3 / 4}\left(1-8 \alpha \mathrm{r}^{2} / 3+16 \alpha^{2} \mathrm{r}^{4} / 15\right) e^{-a r^{2}} \\
\mathrm{R}_{4 \mathrm{~s}}= & (128 / \pi)^{1 / 4}(35 / 16)^{1 / 2} \alpha^{3 / 4}\left(1-4 \alpha \mathrm{r}^{2}+16 \alpha^{2} \mathrm{r}^{4} / 5\right. \\
& \left.\left.-64 \alpha^{3} \mathrm{r}^{6} / 105\right)\right) e^{-a r^{2}} \\
\mathrm{R}_{1 \mathrm{p}}= & (128 / \pi)^{1 / 4}(4 / 3)^{1 / 2} \alpha^{5 / 4} \mathrm{r} e^{-a r^{2}} \\
\mathrm{R}_{2 \mathrm{p}}= & (128 / \pi)^{1 / 4}(10 / 3)^{1 / 2} \alpha^{5 / 4} \mathrm{r}\left(1-4 \alpha \mathrm{r}^{2} / 5\right) e^{-a r^{2}} \\
\mathrm{R}_{3 \mathrm{p}}= & (128 / \pi)^{1 / 4}(70 / 12)^{1 / 2} \alpha^{5 / 4} \mathrm{r}\left(1-8 \alpha \mathrm{r}^{2} / 5\right. \\
& \left.+16 \alpha^{2} \mathrm{r}^{4} / 35\right) e^{-a r^{2}} \\
\mathrm{R}_{1 \mathrm{~d}}= & (128 / \pi)^{1 / 4}(16 / 15)^{1 / 2} \alpha^{7 / 4} \mathrm{r}^{2} e^{-a r^{2}} \\
\mathrm{R}_{2 \mathrm{~d}}= & (128 / \pi)^{1 / 4}(56 / 15)^{1 / 2} \alpha^{7 / 4} \mathrm{r}^{2}\left(1-4 \alpha \mathrm{r}^{2} / 7\right) e^{-a r^{2}} \\
\mathrm{R}_{3 \mathrm{~d}}= & (128 / \pi)^{1 / 4}(252 / 30)^{1 / 2} \alpha^{7 / 4} \mathrm{r}^{2}\left(1-8 \alpha \mathrm{r}^{2} / 7\right.
\end{aligned}
$$

$$
\begin{aligned}
& \left.+16 \alpha^{2} \mathrm{r}^{4} / 63\right) e^{-a r^{2}} \\
\mathrm{R}_{1 \mathrm{f}}= & (128 / \pi)^{1 / 4}(64 / 105)^{1 / 2} \alpha^{9 / 4} \mathrm{r}^{3} e^{-a r^{2}} \\
\mathrm{R}_{2 \mathrm{f}}= & (128 / \pi)^{1 / 4}(288 / 105)^{1 / 2} \alpha^{9 / 4} \mathrm{r}^{3}\left(1-4 \alpha \mathrm{r}^{2} / 9\right) e^{-a r^{2}} \\
\mathrm{R}_{1 \mathrm{~g}}= & (128 / \pi)^{1 / 4}(256 / 945)^{1 / 2} \alpha^{11 / 4} \mathrm{r}^{4} e^{-a r^{2}} \\
\mathrm{R}_{2 \mathrm{~g}}= & (128 / \pi)^{1 / 4}(1408 / 945)^{1 / 2} \alpha^{11 / 4} \mathrm{r}^{4}\left(1-4 \alpha \mathrm{r}^{2} / 11\right) e^{-a r^{2}} \\
\mathrm{R}_{1 \mathrm{~h}}= & (128 / \pi)^{1 / 4}(1024 / 10395)^{1 / 2} \alpha^{13 / 4} \mathrm{r}^{5} e^{-a r^{2}} \\
\mathrm{R}_{1 \mathrm{i}}= & (128 / \pi)^{1 / 4}(4096 / 135135)^{1 / 2} \alpha^{15 / 4} \mathrm{r}^{6} e^{-a r^{2}} \\
\mathrm{R}_{1 \mathrm{j}}= & (128 / \pi)^{1 / 4}(16384 / 2027025)^{1 / 2} \alpha^{17 / 4} \mathrm{r}^{7} e^{-a r^{2}}
\end{aligned}
$$

As a test, all above wave functions have been checked for orthonormality.

Due to the fact that in the model $\hbar \omega$ is different for the different shells, the wave functions with the same orbital angular momentum quantum number $\ell$ are not orthogonal. For these wave functions Gram-Smidth's technique is applied [9].

It is considered instructive to give some relevant guide equations below to facilitate this orthogonalization.

$$
\begin{aligned}
\mathrm{E}_{2}\left(\alpha_{1}, \alpha_{2}, \mathrm{r}\right)=\mathrm{N}_{2} & \left(\alpha_{1}, \alpha_{2}\right)\left[\mathrm{R}_{2}\left(\alpha_{2}, \mathrm{r}\right)+\mathrm{b}_{21}\left(\alpha_{1}, \alpha_{2}\right) \mathrm{R}_{1}\left(\alpha_{1}, \mathrm{r}\right)\right] \\
\mathrm{E}_{3}\left(\alpha_{1}, \alpha_{2}, \alpha_{3}, \mathrm{r}\right)= & \mathrm{N}_{3}\left(\alpha_{1}, \alpha_{2}, \alpha_{3}\right)\left[\mathrm{R}_{3}\left(\alpha_{3}, \mathrm{r}\right)\right. \\
& +\mathrm{b}_{32}\left(\alpha_{1}, \alpha_{2}, \alpha_{3}\right) \mathrm{E}_{2}\left(\alpha_{1}, \alpha_{2}, \mathrm{r}\right) \\
& \left.+\mathrm{b}_{31}\left(\alpha_{1}, \alpha_{3}\right) \mathrm{R}_{1}\left(\alpha_{1}, \mathrm{r}\right)\right] \\
\mathrm{E}_{4}\left(\alpha_{1}, \alpha_{2}, \alpha_{3}, \alpha_{4}, \mathrm{r}\right)= & \mathrm{N}_{4}\left(\alpha_{1}, \alpha_{2}, \alpha_{3}, \alpha_{4}\right)\left[\mathrm{R}_{4}\left(\alpha_{4}, \mathrm{r}\right)\right. \\
& +\mathrm{b}_{43}\left(\alpha_{1}, \alpha_{2}, \alpha_{3}, \alpha_{4}\right) \mathrm{E}_{3}\left(\alpha_{1}, \alpha_{2}, \alpha_{3}, \mathrm{r}\right) \\
& +\mathrm{b}_{42}\left(\alpha_{1}, \alpha_{2} \cdot \alpha_{4}\right) \mathrm{E}_{2}\left(\alpha_{1}, \alpha_{2}, \mathrm{r}\right) \\
& \left.+\mathrm{b}_{41}\left(\alpha_{1}, \alpha_{4}\right) \mathrm{R}_{1}\left(\alpha_{1}, \mathrm{r}\right)\right]
\end{aligned}
$$

where

$$
\begin{aligned}
& \mathrm{N}_{2}\left(\alpha_{1}, \alpha_{2}\right)=\left[\begin{array}{ll}
1-\mathrm{b}^{21} & \left(\alpha_{1}, \alpha_{2}\right)
\end{array}\right]^{-1 / 2} \\
& \mathrm{~N}_{3}\left(\alpha_{1}, \alpha_{2}, \alpha_{3}\right)=\left[1-\mathrm{b}^{32}\left(\alpha_{1}, \alpha_{2}, \alpha_{3}\right)-\mathrm{b}^{31}\left(\alpha_{1}, \alpha_{3}\right)\right]^{-1 / 2} \\
& \mathrm{~N}_{4}\left(\alpha_{1}, \alpha_{2}, \alpha_{3}, \alpha_{4}\right)
\end{aligned}
$$

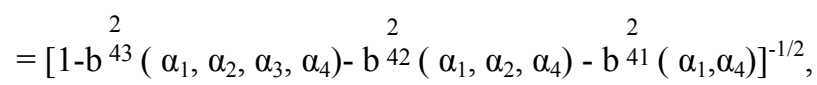

and

$$
\begin{aligned}
& b_{21}\left(\alpha_{1}, \alpha_{2}\right)=-\int_{0}^{\infty} R_{1}\left(\alpha_{1}, r\right) R_{2}\left(\alpha_{2}, r\right) r^{2} d r \\
& b_{32}\left(\alpha_{1}, \alpha_{2}, \alpha_{3}\right)=-\int_{0}^{\infty} E_{2}\left(\alpha_{1}, \alpha_{2}, r\right) R_{3}\left(\alpha_{3}, r\right) r^{2} d r \\
& b_{31}\left(\alpha_{1}, \alpha_{3}\right)=-\int_{0}^{\infty} R_{1}\left(\alpha_{1}, r\right) R_{3}\left(\alpha_{3}, r\right) r^{2} d r \\
& b_{43}\left(\alpha_{1}, \alpha_{2}, \alpha_{3}, \alpha_{4}\right)=-\int_{0}^{\infty} E_{3}\left(\alpha_{1}, \alpha_{2}, \alpha_{3}, r\right) R_{4}\left(\alpha_{4}, r\right) r^{2} d r \\
& b_{42}\left(\alpha_{1}, \alpha_{2}, \alpha_{4}\right)=-\int_{0}^{\infty} E_{2}\left(\alpha_{1}, \alpha_{2}, r\right) R_{4}\left(\alpha_{4}, r\right) r^{2} d r \\
& \mathrm{~b}_{41}\left(\alpha_{1}, \alpha_{4}\right)=-\int_{0}^{\infty} \mathrm{R}_{1}\left(\alpha_{1}, r\right) \mathrm{R}_{4}\left(\alpha_{4}, \mathrm{r}\right) \mathrm{r}^{2} \mathrm{dr}
\end{aligned}
$$


Also, due to the fact that the model requires the orthogonalized wave functions to reproduce the relevant average radii of polyhedral shells given in Fig.1, some additional instructive equations are also given below for radii.

$$
\begin{aligned}
& <\mathrm{r}^{2}>_{2}\left(\alpha_{1}, \alpha_{2}\right)=\int_{0}^{\infty} \mathrm{E}_{2}^{2}\left(\alpha_{1}, \alpha_{2}, \mathrm{r}\right) \mathrm{r}^{4} \mathrm{dr} \\
& <\mathrm{r}^{2}>_{3}\left(\alpha_{1}, \alpha_{2}, \alpha_{3}\right)=\int_{0}^{\infty} \mathrm{E}_{3}^{2}\left(\alpha_{1}, \alpha_{2}, \alpha_{3}, \mathrm{r}\right) \mathrm{r}^{4} \mathrm{dr} \\
& <\mathrm{r}^{2}>_{4}\left(\alpha_{1}, \alpha_{2}, \alpha_{3}, \alpha_{4}\right)=\int_{0}^{\infty} \mathrm{E}_{4}^{2}\left(\alpha_{1}, \alpha_{2}, \alpha_{3}, \alpha_{4}, \mathrm{r}\right) \mathrm{r}^{4} \mathrm{dr}
\end{aligned}
$$

While is known that $\alpha_{1}=\left(\mathrm{m} / 2 \hbar^{2}\right) * \hbar \omega_{1}$, in the case of wave functions which need orthogonalization $\alpha_{2}, \alpha_{3}$, and $\alpha_{4}$ in the model are determined as follows. For example for the case of $2 p$ state for neutrons, while $\alpha_{1}$ is determined from Eq.(5) by using the radius $3.568 \mathrm{fm}$ (see N3 in Figure 1), the parameter $\alpha_{2}$ in the first equation of (12) must take on a value which leads to the radius $5.0372 \mathrm{fm}$ (see N4 in Figure 1). For the case of $3 \mathrm{p}$ state for neutrons, the parameters $\alpha_{1}$ and $\alpha_{2}$ in the second equation of (12) take on the previous values, while $\alpha_{3}$ must take on a value which leads to the radius $7.8688 \mathrm{fm}$ (see N11 in Figure 1).

\subsubsection{Binding Energies}

As known, the binding energy for each quantum state in a harmonic oscillator potential is given by Eq.(13):

$$
\mathrm{E}_{\mathrm{B}}=\mathrm{v}-\hbar \omega(\mathrm{n}+3 / 2) \text {. }
$$

As aforementioned, in Eq.(13) all $\hbar \omega$ come from Eq.(5) (with respect to the only two size parameters $r_{p}$ and $r_{n}$ ) and all potential depths $\mathrm{v}$ come from Eq.(6) (with respect

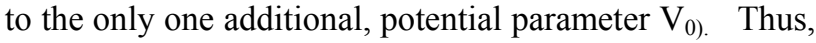
$\mathrm{E}_{\mathrm{B}}$ from Eq.(13) is determined with respect to only 3 universal parameters.

Now, since the nuclear problem basically refers to two-body forces, in order to avoid the double counting, the potential portion of the second part of Eq.(13) should be divided by two. Further, since according to the Virial theorem half of the quantity $\hbar \omega(n+3 / 2)$ is potential energy and half is kinetic energy, Eq.(13) takes the form of Eq.(14)

$$
\begin{aligned}
\mathrm{E}_{\mathrm{B}} & =1 / 2[\mathrm{v}-1 / 2 \hbar \omega(\mathrm{n}+3 / 2)]-1 / 2 \hbar \omega(\mathrm{n}+3 / 2)]) \\
& =1 / 2 \mathrm{v}-3 / 4 \hbar \omega(\mathrm{n}+3 / 2)
\end{aligned}
$$

Given that the potential v, according to Eq.(6), is

$$
\mathrm{v}=\mathrm{V}_{0}+\hbar \omega(\mathrm{n}+3 / 2) \text {, }
$$

the final expression of binding energy for each proton or neutron state takes the form of Eq.(16).

$$
\begin{aligned}
\left(\mathrm{E}_{\mathrm{B}}\right)_{1} & =1 / 2\left[\mathrm{~V}_{0}+\hbar \omega(\mathrm{n}+3 / 2]-3 / 4 \hbar \omega(\mathrm{n}+3 / 2)\right. \\
& =1 / 2 \mathrm{~V}_{0}-1 / 4 \hbar \omega_{1}\left(\mathrm{n}_{1}+3 / 2\right)
\end{aligned}
$$

The index 1 in Eq.(16) refers to all states where the orbital angular momentum quantum number $\ell$ appears for the first time.

However, for a second, a third and a fourth appearance of a state with the same $\ell$, in the place of the quantity $\hbar \omega$ $(n+3 / 2)$ in Eq.(16) we should take the corresponding quantity due to the necessary orthogonalization. Thus, based on Eqs. (9) - (11), we get

$$
\begin{aligned}
\left(\mathrm{E}_{\mathrm{B}}\right)_{2}= & 1 / 2 \mathrm{~V}_{0}-1 / 4 \mathrm{~N}_{2}\left[\hbar \omega_{2} *\left(\mathrm{n}_{2}+3 / 2\right)\right. \\
& \left.+\mathrm{b}_{21} * \hbar \omega_{1}\left(\mathrm{n}_{1}+3 / 2\right)\right] \\
\left(\mathrm{E}_{\mathrm{B}}\right)_{3}= & 1 / 2 \mathrm{~V}_{0}-1 / 4 \mathrm{~N}_{3}\left\{\hbar \omega_{3} *\left(\mathrm{n}_{3}+3 / 2\right)\right. \\
& \left.+\mathrm{b}_{32} * 4\left[-\left(\mathrm{E}_{\mathrm{B}}\right)_{2}+1 / 2 \mathrm{~V}_{0}\right]+\mathrm{b}_{31} * \hbar \omega_{1}\left(\mathrm{n}_{1}+3 / 2\right)\right\} \\
\left(\mathrm{E}_{\mathrm{B}}\right)_{4}= & 1 / 2 \mathrm{~V}_{0}-1 / 4 \mathrm{~N}_{4}\left\{\hbar \omega_{4} *\left(\mathrm{n}_{4}+3 / 2\right)\right. \\
& +\mathrm{b}_{43} * 4\left[-\left(\mathrm{E}_{\mathrm{B}}\right)_{3}+1 / 2 \mathrm{~V}_{0}\right] \\
& \left.+\mathrm{b}_{42} * 4\left[-\left(\mathrm{E}_{\mathrm{B}}\right)_{2}+1 / 2 \mathrm{~V}_{0}\right]+\mathrm{b}_{41} * \hbar \omega_{1}\left(\mathrm{n}_{1}+3 / 2\right)\right\}
\end{aligned}
$$

Finally, the total binding energy of a nucleus in the model is given by Eq.(18)

$$
\left(\mathrm{E}_{\mathrm{B}}\right)_{\mathrm{total}}=\Sigma_{i=1}^{A}\left(\mathrm{E}_{\mathrm{B}}\right)_{\mathrm{i}}+\Sigma_{i=1}^{A} \mathrm{~V}_{\mathrm{LiSi}}-\sum_{i \neq j}^{Z} \mathrm{E}_{\mathrm{Cij}},
$$

where: the spin-orbit term is given [8] by Eq.(19)

$$
\begin{aligned}
\sum_{i=1}^{A} \mathrm{~V}_{\mathrm{LiSi}} & =\lambda \Sigma_{i=1}^{A}[\mathrm{i} / \mathrm{r} * \mathrm{dV}(\mathrm{r}) / \mathrm{dr}] * \boldsymbol{\ell}_{\mathrm{i}} \mathbf{s}_{\mathbf{i}} \\
& =\lambda \Sigma_{i=1}^{A}\left(\hbar \omega_{\mathrm{i}}\right)^{2} /\left(\hbar^{2} / \mathrm{m}\right) * \boldsymbol{\ell}_{\mathrm{i}} \mathbf{s}_{\mathbf{i}},
\end{aligned}
$$

with $\lambda=0.03$ (This is the fourth and the last universal parameter of the present model), and the Coulomb term is given by Eq.(20):

$$
\Sigma_{i \neq j}^{Z} E_{\mathrm{Cij}}=\Sigma_{i \neq j}^{Z} \mathrm{e}^{2} / \mathrm{d}_{\mathrm{ij}} .
$$

The distance $\mathrm{d}_{\mathrm{ij}}$ between any two proton average positions in Figure $\mathbf{1}$ is calculated from the coordinates of the proton polyhedral vertices (standing for the proton average positions) in this figure (see section 2.2).

An extra term in Eq.(18) due to isospin is not needed since the isospin is here taken care of by the different average shell structure between protons and neutrons as apparent from Figrue 1.

In Table 3 the derived energies (including spin-orbit) for all proton and neutron single-particle states up to ${ }^{208} \mathrm{~Pb}$ are given. Specifically, in col.2 of the table for each state (col.1) the numerical value of energy for protons and underneath for neutrons are listed. In col. 4 the energy of all nucleons in a state (col. 3) is given again in two rows for each state as before. In the remaining cols. 5-11 the energies from col. 4 relevant to ${ }^{40} \mathrm{Ca},{ }^{48} \mathrm{Ca},{ }^{54} \mathrm{Fe}$, ${ }^{90} \mathrm{Zr},{ }^{108} \mathrm{Sn},{ }^{114} \mathrm{Te},{ }^{142} \mathrm{Nd}$, and ${ }^{208} \mathrm{~Pb}$ are repeated. There, one can also see which states are considered in the framework of the model occupied by protons and neutrons in the structure of these nuclei.

For all seven nuclei examined ${ }^{16} \mathrm{O}$ is taken as a core. This means that the ${ }^{16} \mathrm{O}$ experimental binding energy 
(127.62 MeV) and its Coulomb energy (11.98 MeV derived from the model) are assumed known. That is, the energy for the ${ }^{16} \mathrm{O}$ core is taken equal to $139.60 \mathrm{MeV}$ and is listed in the fourth row from the bottom of Table 3. In the following row of the table the calculated relevant Coulomb energies in the framework of the model are given and underneath, the predicted and experimental binding energies [10]. The agreements between these two binding energies are apparent and quite striking. This agreement is further strengthened by the fact that the model employed uses only four universal parameters.
Namely, these parameters are: The two size parameters $r_{p}$ $=0.860 \mathrm{fm}$ and $r_{n}=0.974 \mathrm{fm}$, the one potential parameter $\mathrm{V}_{0}=40.268 \mathrm{MeV}$, and the one spin-orbit parameter $\lambda$ $=0.03$.

As obvious from Table 3 the binding energy of any nucleus examined is obtained by summing up the energies of the single particle states involved in this nucleus. This means that the model employed here considers exclusively an independent particle motion for all nucleons. In other words, the model considers that for each closed shell we have a complete saturation of nuclear forces.

\begin{tabular}{|c|c|c|c|c|c|c|c|c|c|c|c|}
\hline State & Energy & \# & Total & ${ }^{40} \mathrm{Ca}$ & ${ }^{48} \mathrm{Ca}$ & ${ }^{54} \mathrm{Fe}$ & ${ }^{90} \mathrm{Zr}$ & ${ }^{108} \mathrm{Sn}$ & ${ }^{114} \mathrm{Te}$ & ${ }^{142} \mathrm{Nd}$ & ${ }^{208} \mathrm{~Pb}$ \\
\hline \multirow{2}{*}{$1 \mathrm{~d} 5 /$} & 12.035 & 6 & 72.21 & 72.21 & 72.21 & 72.21 & 72.21 & 72.21 & 72.21 & 72.21 & 72.21 \\
\hline & 10.258 & & 61.55 & 61.55 & 61.55 & 61.55 & 61.55 & 61.55 & 61.55 & 61.55 & 61.55 \\
\hline \multirow[t]{2}{*}{$1 \mathrm{~d} 3 /$} & 11.878 & 4 & 47.51 & 47.51 & 47.51 & 47.51 & 47.51 & 47.51 & 47.51 & 47.51 & 47.51 \\
\hline & 10.023 & & 40.09 & 40.09 & 40.09 & 40.09 & 40.09 & 40.09 & 40.09 & 40.09 & 40.09 \\
\hline \multirow[t]{2}{*}{$2 \mathrm{~s} 1 /$} & 12.476 & 2 & 24.95 & 24.95 & 24.95 & 24.95 & 24.95 & 24.95 & 24.95 & 24.95 & 24.95 \\
\hline & 10.606 & & 21.21 & 21.21 & 21.21 & 21.21 & 21.21 & 21.21 & 21.21 & 21.21 & 21.21 \\
\hline \multirow[t]{2}{*}{$1 \mathrm{f} 7 /$} & 12.452 & 8 & 99.62 & & - & 74.72 & 99.62 & 99.62 & 99.62 & 99.62 & 99.62 \\
\hline & 11.924 & & 95.39 & & 74.21 & 95.39 & 95.39 & 95.39 & 95.39 & 95.39 & 95.39 \\
\hline \multirow[t]{2}{*}{$1 \mathrm{f} 5 /$} & 12.332 & 6 & 73.99 & & & & 73.99 & 73.99 & 73.99 & 73.99 & 73.99 \\
\hline & 11.787 & & 70.72 & & & & 70.72 & 70.72 & 56.72 & 56.72 & 56.72 \\
\hline \multirow[t]{2}{*}{$2 \mathrm{p} 3 /$} & 16.263 & 4 & 65.05 & & & & 65.05 & 65.05 & 65.05 & 65.05 & 65.05 \\
\hline & 16.285 & & 65.14 & & & & 65.14 & 65.14 & 65.14 & 65.14 & 65.14 \\
\hline \multirow[t]{2}{*}{$2 \mathrm{p} 1 /$} & 10.16 & 2 & 20.32 & & & & 20.32 & 20.32 & 20.32 & 20.32 & 20.32 \\
\hline & 16.227 & & 32.45 & & & & 32.45 & 32.45 & 32.45 & 32.45 & 32.45 \\
\hline \multirow[t]{2}{*}{ 1g9/ } & 9.904 & 10 & 99.04 & & & & - & 99.04 & 99.04 & 99.04 & 99.04 \\
\hline & 7.898 & & 78.98 & & & & 78.98 & 78.98 & 78.98 & 78.98 & 78.98 \\
\hline \multirow[t]{2}{*}{ 1g7/ } & 9.721 & 8 & 77.77 & & & & & - & - & - & 77.77 \\
\hline & 7.635 & & 61.08 & & & & & - & - & 61.08 & 61.08 \\
\hline \multirow[t]{2}{*}{$2 \mathrm{~d} 5 /$} & 14.373 & 6 & 86.24 & & & & & - & 30.35 & 91.06 & 86.24 \\
\hline & 17.29 & & 103.74 & & & & & 103.74 & 103.74 & 103.74 & 103.74 \\
\hline \multirow[t]{2}{*}{$2 \mathrm{~d} 3 /$} & 15.117 & 4 & 60.47 & & & & & - & - & 60.47 & 60.47 \\
\hline & 17.267 & & 69.07 & & & & & 34.54 & 69.07 & 69.07 & 69.07 \\
\hline \multirow[t]{2}{*}{$3 \mathrm{~s} 1 /$} & 12.574 & 2 & 25.15 & & & & & & - & - & 25.15 \\
\hline & 10.842 & & 21.68 & & & & & & 21.68 & 21.68 & 21.68 \\
\hline \multirow[t]{2}{*}{ 1h11/ } & 10.909 & 12 & 130.91 & & & & & & & - & 130.91 \\
\hline & 11.084 & & 133.01 & & & & & & & 133.01 & 133.01 \\
\hline \multirow[t]{2}{*}{ 1h9/ } & 12.602 & 10 & 126.02 & & & & & & & & - \\
\hline & 10.445 & & 104.45 & & & & & & & & 104.45 \\
\hline \multirow[t]{2}{*}{$2 \mathrm{f} 7 /$} & - & 8 & & & & & & & & & - \\
\hline & 16.311 & & 130.49 & & & & & & & & 130.49 \\
\hline \multirow[t]{2}{*}{$2 \mathrm{f5} /$} & - & 6 & & & & & & & & & - \\
\hline & 16.269 & & 97.61 & & & & & & & & 97.61 \\
\hline \multirow[t]{2}{*}{$3 \mathrm{p} 3 /$} & - & 4 & & & & & & & & & - \\
\hline & 17.687 & & 70.75 & & & & & & & & 70.75 \\
\hline \multirow[t]{2}{*}{$3 \mathrm{p} 1 /$} & - & 2 & - & & & & & & & & - \\
\hline & 17.644 & & 35.29 & & & & & & & & 35.29 \\
\hline \multirow[t]{2}{*}{ 1i13/ } & - & 14 & - & & & & & & & & - \\
\hline & 7.436 & & 104.1 & & & & & & & & 104.1 \\
\hline${ }^{16} \mathrm{O}$ core & & & & 139.61 & 139.61 & 139.61 & 139.61 & 139.61 & 139.61 & 139.61 & 139.61 \\
\hline $\mathrm{E}_{\mathrm{C}}$ & & & & -64.81 & -64.81 & -105.92 & -225.8 & -330.9 & -357.53 & -448.79 & -769.24 \\
\hline $\mathrm{E}_{\mathrm{B}} \bmod .$. & & & & 342.31 & 416.52 & 471.31 & 782.98 & 915.18 & 961.13 & 1185.14 & 1636.4 \\
\hline$E_{B}$ exp. & & & & 342.06 & 416.01 & 471.77 & 783.24 & 914.66 & 961.23 & 1185.17 & 1636.5 \\
\hline
\end{tabular}


It is necessary to explain why in Table 3 the binding energy of neutron $1 \mathrm{f} 5 / 2$ state for ${ }^{114} \mathrm{Te},{ }^{142} \mathrm{Nd}$, and ${ }^{208} \mathrm{~Pb}$ is different than that for ${ }^{90} \mathrm{Zr}$ and ${ }^{108} \mathrm{Sn}$. For the nuclei of the table up to ${ }^{108} \mathrm{Sn}$ the six neutrons of N4 are at the vertices of N5 marked with $h$ and the binding energy of neutron $1 \mathrm{f} 5 / 2$ state is estimated as part of N5.. For the heavier nuclei the $\mathrm{N} 4$ is completed and the binding energy of neutron $1 \mathrm{f} 5 / 2$ state (shown in bold letters in the table) is estimated as part of this polyhedron.

In Table 3 some additional binding energies are shown in bold letters. For these states some discussion is needed as for the $1 \mathrm{f} 5 / 2$ neutron state above. Specifically, in ${ }^{108} \mathrm{Sn}$ the energy $34.54 \mathrm{MeV}$ of the neutron $2 \mathrm{~d} 3 / 2$ state corresponds to two neutrons and, of course, should be half the energy $69.08 \mathrm{MeV}$ in column 4 (which corresponds to four neutrons). An identical explanation is valid for the difference of energies $30.24 \mathrm{MeV}$ and 60.48 $\mathrm{MeV}$ for the proton $2 \mathrm{~d} 3 / 2$ state in the case of ${ }^{114} \mathrm{Te}$. In the case of ${ }^{142} \mathrm{Nd}$ the energy $91.08 \mathrm{MeV}$ of the $2 \mathrm{~d} 5 / 2$ protons is calculated assuming that the six neutrons are on $\mathrm{Z} 8$ instead of on Z7. The Z7 is completed later, after the formation of N5.

Some extra discussion is also required for the $1 \mathrm{f} 7 /$ neutron state of ${ }^{48} \mathrm{Ca}$. The state binding energy shown in bold numbers in Table 3 is derived by assuming that the eight neutrons of this state fill the polyhedron Z4 which for all other seven nuclei of Table $\mathbf{3}$ is filled by protons. This is permissible according to the assumptions of the model as long this polyhedron is completely empty. The assignment of polyhedra as proton or neutron polyhedra in Figure 1 assumes that all polyhedral shells are filled as for example in the case of ${ }^{208} \mathrm{~Pb}$. In the cases where some polyhedra are completely empty, one can consider that these polyhedra are available either for protons and or for neutrons. In the case of ${ }^{48} \mathrm{Ca}$ the radius of $\mathrm{Z4}$ is derived from that of $Z 2$, i.e., $R_{Z 4}($ prime $)=2.541+r_{p}+r_{n}$ $=4.375 \mathrm{fm}$, while previously it was $\mathrm{R}_{\mathrm{Z} 4}=2.541+2 \mathrm{r}_{\mathrm{p}}=$ $4.341 \mathrm{fm}$. This value is smaller than the value of $\mathrm{R}_{\mathrm{N} 4}=$ $4.459 \mathrm{fm}$ and thus preferable since it leads to larger binding energy. In general, in the case of valence protons occupying a neutron polyhedron or vice versa Eq.(3) is again applied with the proper choice of $\mathrm{d}_{12}$ as explained in section 2.2.

\subsubsection{Mean Radii}

Since the wave functions are known in the framework of the model (section 2.3), the nuclear radii can be calculated straightforwardly by using these functions. However, due to the way these wave functions have been correlated with the size of nuclear shells via Eq.(5), average radii can equivalently be calculated by using simple formulae, as seen below.

Average charge radius:

$$
<\mathrm{r}^{2}>_{\mathrm{ch}}=\Sigma_{i=1}^{Z} \mathrm{r}_{i}^{2} / \mathrm{Z}+\mathrm{r}_{\text {ch..proton }}^{2}-\mathrm{r}_{\text {ch.neutron }}^{2} * \mathrm{~N} / \mathrm{Z}
$$

where $r_{\text {ch.proton }}=r_{p}=0.842 \mathrm{fm}$ and $r_{\text {ch.neutron }}=0.34 \mathrm{fm}[7]$.

Average neutron radius:

$$
<\mathrm{r}^{2}>_{\mathrm{n}}=\Sigma_{i=1}^{N} \mathrm{r}_{i}^{2} / \mathrm{N}+\mathrm{r}_{\text {neutron }}^{2},
$$

where $\mathrm{r}_{\text {neutron }}=\mathrm{r}_{\mathrm{n}}=0.974 \mathrm{fm}$.

Average mass radius:

$$
<\mathrm{r}^{2}>_{\mathrm{m}}=\left[\Sigma_{i=1}^{A} \mathrm{r}_{\mathrm{i}}^{2}+\mathrm{Z}^{*} \mathrm{r}_{p}^{2}+\mathrm{N}^{*} \mathrm{r}_{n}^{2}\right] / \mathrm{A}
$$

If point radii are required, in the above formulae only the first term in each equation remains. All values of $r_{i}$ needed are included in Figure 1 (See right corner at the bottom of each block).

In Table 4 the predictions of the present model for radii are given for all eight nuclei used as a sample in the present manuscript. In this table the predictions of the model on average charge, on average neutron and on average mass radii are given together with the difference between proton and neutron radii. The comparisons of the model predictions for charge radii with the relevant experimental data (coming from [11] and only for ${ }^{54} \mathrm{Fe}$ from [12] which is a model prediction), in all cases, are very good. In most of the cases our predictions and the data are identical. For ${ }^{114} \mathrm{Te}$ an experimental value has not been found. However, the comparisons of the model predictions with the available experimental data for the point neutron-proton radii are good only for the first five nuclei of the table. For the next two nuclei, i.e. ${ }^{114} \mathrm{Te}$ and ${ }^{142} \mathrm{Nd}$, experimental values have not been found and for the last nucleus ${ }^{208} \mathrm{~Pb}$ the comparison with experiments is problematic. For that nucleus the relevant pieces of information from the literature concerning neutron-proton radii are given below together with comments for the other nuclei.

From [13] for ${ }^{40} \mathrm{Ca}$ we have neutron-proton radius $-0.20 \mathrm{fm}$ till $-0.40 \mathrm{fm}$ and in Table 4 we write the average of these two values, i.e., $-0.30 \mathrm{fm}$. The value $0.12 \mathrm{fm}$ for ${ }^{48} \mathrm{Ca}$ is taken from [14]. From [15] we have the values for ${ }^{54} \mathrm{Fe} \quad 0.04_{-0.08}^{+0.06} \mathrm{fm}$ and for ${ }^{90} \mathrm{Zr} 0.09 \pm 0.02 \mathrm{fm}$ and in Table 4 we write -0.04 and 0.09 , respectively. The value $0.11 \mathrm{fm}$ for ${ }^{108} \mathrm{Sn}$ in the table is estimated from the neutron rms radius 4.615 fm from [16] minus our point proton rms radius $4.50 \mathrm{fm}$. Finally, the value $0.55 \mathrm{fm}$ in Table 4 for ${ }^{208} \mathrm{~Pb}$ is estimated by employing the ratio of neutron radius/ proton radius equal to $1.07 \pm 0.03 \mathrm{fm}$ from [17] and our point proton rms radius $5.46 \mathrm{fm}$, i.e., $1.1 * 5.46-5.46=0.55 \mathrm{fm}$. Other data somehow supporting our value $0.55 \mathrm{fm}$ in Table 4 comes from [18] where the neutron radius is $6.7 \mathrm{fm}$, from [19] where this radius is $5.99 \pm 0.10 \mathrm{fm}$, and from [20] where $r_{n}-r_{p}=0.33 \pm$ $0.17 \mathrm{fm}$. There is a great variety of experimental and theoretical results for the neutron-proton rms radius of ${ }^{208} \mathrm{~Pb}$ varying from small to large values. In general, the estimation of neutron rms radius of a nucleus is very difficult task. The opposite is true for the estimation of proton rms radius of a nucleus, due to the charge of protons. 
Table 4. Average charge, neutron, point neutron-proton, and mass radi .

\begin{tabular}{|c|c|c|c|c|c|c|c|c|}
\hline Nucleus & ${ }^{40} \mathrm{Ca}$ & ${ }^{48} \mathrm{Ca}$ & ${ }^{54} \mathrm{Fe}$ & ${ }^{90} \mathrm{Zr}$ & ${ }^{108} \mathrm{Sn}$ & ${ }^{114} \mathrm{Te}$ & ${ }^{142} \mathrm{Nd}$ & ${ }^{208} \mathrm{~Pb}$ \\
\hline Polyh.:Radius & & & & Occup & ation & & & \\
\hline Z1: 1.554 & 2 & 2 & 2 & 2 & 2 & 2 & 2 & 2 \\
\hline Z2: 2.541 & 6 & 6 & 6 & 6 & 6 & 6 & 6 & 6 \\
\hline Z3: 3.946 & 12 & 12 & 12 & 12 & 12 & 12 & 12 & 12 \\
\hline Z4: 4.341 & & 8 & 6 & 8 & 8 & 8 & 8 & 8 \\
\hline Z5: $\quad 5.2122$ & & & & 12 & 12 & 12 & 12 & 12 \\
\hline Z6: 5.5167 & & & & & 10 & 12 & 12 & 12 \\
\hline Z7: 6.293 & & & & & & & 6 & 6 \\
\hline Z8: 6.8712 & & & & & & & 2 & 24 \\
\hline N1: 0.974 & 2 & 2 & 2 & 2 & 2 & 2 & 2 & 2 \\
\hline N2: 2.511 & 6 & 6 & 6 & 6 & 6 & 6 & 6 & 6 \\
\hline N3: 3.568 & 12 & 12 & 12 & 12 & 12 & 12 & 12 & 12 \\
\hline N4: 5.0372 & & & 2 & 24 & 24 & 24 & 24 & 24 \\
\hline N5: 4.459 & & & 6 & 6 & 6 & 6 & 6 & 6 \\
\hline N6: 6.6713 & & & & & & 4 & 12 & 12 \\
\hline N7: 6.175 & & & & & 8 & 8 & 8 & 8 \\
\hline N8: 6.9329 & & & & & & & 12 & 12 \\
\hline N9: 8.123 & & & & & & & & 8 \\
\hline N10: 6.748 & & & & & & & & 24 \\
\hline N11: 7.8688 & & & & & & & & 12 \\
\hline $\mathrm{ch}<\mathrm{r}^{2}>^{1 / 2} \mathrm{mod}$ & 3.48 & 3.48 & 3.72 & 4.27 & 4.56 & 4.61 & 4.9 & 5.51 \\
\hline$c h<r^{2}>^{1 / 2} \exp$. & 3.48 & 3.48 & 3.69 & 4.27 & 4.56 & & 4.91 & 5.51 \\
\hline$n_{n}<r^{2}>^{1 / 2}$ mod. & 3.25 & 3.65 & 3.73 & 4.4 & 4.7 & 4.86 & 5.43 & 6.18 \\
\hline$\left.{ }_{n}<r^{2}>^{1 / 2}{ }_{c h}<r^{2}>^{1 / 2}\right]$ mod. & -0.29 & 0.12 & -0.03 & 0.09 & 0.1 & 0.22 & 0.5 & 0.64 \\
\hline$\left[{ }_{n}<r^{2}>^{1 / 2}{ }_{c h}<r^{2}>^{1 / 2}\right] \exp$ & -0.3 & 0.12 & -0.04 & 0.09 & 0.11 & - & - & 0.55 \\
\hline $\mathrm{m}^{<\mathrm{r}^{2}>^{1 / 2} \mathrm{mod}}$ & 3.38 & 3.56 & 3.81 & 4.35 & 4.69 & 4.8 & 5.22 & 5.93 \\
\hline
\end{tabular}

It is interesting one to remark the relation between proton and neutron rms radii of a nucleus in the present model. In the model the structures of proton shells and of neutron shells are interweaved (see Figure 1), as a result of basic properties of the constituent particles of a nucleus, i.e., of the fermionic nature of protons and neutrons. Thus, the predictions on neutron rms radii in the present model depend on proton rms radii. The fact that the binding energies of Table 3 and the proton rms radii of Table 4 are in very good agreements with the experimental data gives a special interest to our neutron rms predictions which should be investigated deeper.

\subsubsection{Other Applications of the Model}

The present work follows a standard procedure starting with the Schrödinger equation. Previous versions of the model employing mainly a semi-classical approach (in the spirit of the Ehrenfest theorem [21-23] that for the average values the laws of Classical Mechanics are valid), which uses the same nucleon average positions as those of Figure 1, have already been applied in many cases. Some are $\Sigma$ atoms [24], nuclear momentum and density distribution [25], moments of inertia and rotating spectra [26], excited states [27], neutron nuclei [27], derivation of a two-body potential [28], and nuclear reactions [29].

\section{Conclusions}

The multi-harmonic shell clustering of a nucleus prented here, employing only four universal parameters (2 size parameters $r_{p}$ and $r_{n}, 1$ potential parameter $V_{0}$ and 1 spin-orbit parameter $\lambda$ ) has proved very successful. The predictions on magic and semi-magic numbers, binding energies and radii are very satisfactory. We expect that further applications of the present approach on more observables will be successful as well, as are the cases of application of the semi-classical version of the model (see section 2.3.3 above). The present work apparently offers a link between collective model and independent particle model of nuclear structure. Indeed, the average sizes of shells are derived in the spirit of Independent Particle Model (see section 2.1 above) and these average forms in the spirit of Collective Model are employed to reproduce the rotational spectra [30].

While in the present work a number of nuclei (eight nuclei) are used as an example to demonstrate the model, Figure 1 could be used for the structure of the core of any nucleus.

Perhaps, the most significant conclusion of the present work is that it supports a shell clustering of nuclear 
structure exclusively based on the fermionic nature of nucleons and their average sizes. In brief, the anti-symmetric wave function of nucleons makes them behave as if a repulsive force (of unknown nature) is acting among them. This force for nucleons on spherical shells, in order to obtain equilibrium of forces (which is a necessary condition for an independent particle motion of nucleons), it leads to equilibrium structures for nuclear shells, i.e., to the structures of regular polyhedra and their derivative polyhedra [2, 4-5]. Furthermore, these polyhedral structures are finally closed packed to obtain maximum nuclear density for the average positions of nucleons by taking advantage of their finite sizes.

The basic computer programs employed by the present work are available on request.

\section{Acknowledgements}

The author is deeply indebted to Dr. D. Lenis of our Institute for his excellent computer work related to the present manuscript.

\section{REFERENCES}

[1] C. W. Sherwin, "Introduction to Quantum Mechanics," Holt Rinehart and Winston, New York, 1959, p.205.

[2] J. Leech, "Equilibrium of Sets of Particles on a Sphere," Mathematical Gazette, Vol. 41, No. 36, 1957, pp. 81-90. doi: $10.2307 / 3610579$

[3] G. S. Anagnostatos, "Symmetry Description of the Independent Particle Model," Lettere Al Nuovo Cimento, Vol. 29, No. 6, 1980, pp. 188-192.

[4] G. S. Anagnostatos, "The Geometry of the Quantization of Angular Momentum $(\ell, s, j)$ in Fields of Central Symmetry, Lettere Al Nuovo Cimento, Vol. 28, No. 17, 1980, pp. 573- 576.

[5] G. S. Anagnostatos, J. Giapitzakis, A. Kyritsis, "Rotational Invariance of Orbital-Angular-Momentum Quantization of Direction for Degenerate States", Lettere Al Nuovo Cimento, Vol. 32, No. 11, 1981, pp. 332-335. doi:10.1007/BF02745301

[6] H. M. Cundy and A. P. Rollett, "Mathematical Models," $2^{\text {nd }}$ Ed., Oxford University Press, 1961, p.76.

[7] H. S. M. Coxeter, "Regular Polytopes", $2^{\text {nd }}$ Ed., The Macmillan Company, New York, 1963.

[8] G. S. Anagnostatos, "Isomorphic Shell Model for Closed-Shell Nuclei, " International Journal of Theoretical Physics, Vol. 24, 1985, pp. 579-613. doi:10.1007/BF00670466

[9] De Jager, C. W. H. de Vries, C. de Vries, "Nuclear Charge and Momentum Distribution," Atomic Data and Nuclear Data Tables, Vol. 14, No. 5-6, 1974, pp. 479-665. doi:10.1016/S0092-640X(74)80002-1

[10] W. F. Hornyak, "Nuclear Structure," Academic, New York, 1975, p 13.

[11] J. D. Vergados, "Mathematical Methods in Physics,"
Akourastos Giannis, Greece, 1970.

[12] A. H. Wapstra and N. B. Gove, "Atomic Mass Table," Automic Data and Nuclear Data Tables, Vol. 9, 1971, pp. 267-301. doi:10.1016/S0092-640X(09)80001-6

[13] E.G.Nadjakov, K.P.Marinova, and Yu.P.Gangrsky, "Systematics of Nuclear Charge Radii", Automic Data and Nuclear Data Tables, Vol. 56, 1994, pp. 133-167. doi:10.1006/adnd.1994.1004

[14] I. Angeli, "Recommended Values of R.M.S. Charge Radii”, Heavy Ion Phys., Vol. 8, No. 1-2, 1998, pp. 23-29.

[15] L. Ray, G. W. Hoffmann, and W. R. Coker, "Nonrelativistic and Relativistic Descriptions of Proton-Nucleus Scattering," Physics Reports, Vol. 212, No. 5, 1992, pp. 223-328. doi:10.1016/0370-1573(92)90156-T

[16] R. C. Barrett., "Colloques," Journal de Physique, Vol. 34, 1973, pp. 23-28.

[17] A. Trzcinska, "Nuclear Periphery Studied with Antiprotonic Atoms", Hyp. Inter., Vol. 194, No. 1, 2009, pp. 271-276.

[18] J. Terasaki, J. Engel, "Self-Consistent Description of Multipole Strength: Systematic Calculations," Physical Review C, Vol. 74, No. 4, 2006, pp. 044301-044319. doi:10.1103/PhysRevC.74.044301

[19] S. D. Schery, D. A. Lind and C. D. Zafiratos, "Radius of the Neutron Distribution in ${ }^{208} \mathrm{~Pb}$ from $(\mathrm{p}, \mathrm{n})$ Quasielastic Scattering," Physical Review C, Vol. 9, No. 1, 1974, pp. 416-418. doi:10.1103/PhysRevC.9.416

[20] G. F. Bertsch, P. F. Bortignon, R. A. Broglia, "Damping of Nuclear Excitations," Reviews of Modern Physics, Vol. 55, 1983, pp. 287-314. doi:10.1103/RevModPhys.55.287

[21] H. J. Koemer and J. P. Schiffer, "Neutron Radius of ${ }^{208} \mathrm{~Pb}$ from Sub-Coulomb Pickup," Physical Review Letters, Vol. 27, No. 21, 1971, pp. 1457-1460. doi:10.1103/PhysRevLett.27.1457

[22] S. Abrahamyan, Z. Ahmed, H. Albataineh, K. Aniol. D. S. Armstrong, et al., "Measurement of the Neutron Radius of ${ }^{208} \mathrm{~Pb}$ through Parity-Violation in Electron Scattering", cited as: arXiv: 1201.2568v2 [nucl-ex], Cornell University Library, 13 Jan. 2012, journal reference: Physical Review Letters, Vol. 108, No. 11, 2012, pp. 112502-112507.

[23] E. Merzbacher, "Quantum Mechanics," John Wiley and Sons, Inc. New York, 1961, p. 42

[24] C. Cohen-Tannoudji, B. Diu, F. Laloe, "Quantum Mechanics," John Wiley \& Sons, New York, 1977, p. 240.

[25] A. Bohr, B. R. Mottelson, "Nuclear Structure," W. A. Benjamin, Inc., Advanced Book Program, Reading, Massachusetts, London, Vol. 2, 1975.

[26] J. Dabrowski, J. Rozynek and G. S. Anagnostatos, " $\Sigma$ ' Atoms and the $\Sigma \mathrm{N}$ Interaction," Eur. Phys. J. A, Vol. 14, 2002, pp. 125-131.

[27] G. S. Anagnostatos, A. N. Antonov, P. Ginis, J. Giapitzakis and M. K. Gaidarov, "Nucleon Momentum and Density Distributions in ${ }^{4} \mathrm{He}$ Considering Internal Rotation," Physical Review C, Vol. 58, No. 4, 1998, pp. 
2115-2119.

[28] M. K. Gaidarov, A. N. Antonov, G. S. Anagnostatos, S. E. Massen, M. V. Stoitsov and P. E. Hodgson, "Proton Momentum Distribution in Nuclei beyond ${ }^{4} \mathrm{He}$," Physical Review C, Vol. 52, No. 6, 1995, pp. 3026-3031.

[29] G. S. Anagnostatos, P. Ginis, J. Giapitzakis, “ $\alpha$-Planar States in ${ }^{28} \mathrm{Si}$," Physical Reviewe C, Vol. 58, No. 6, 1998, pp. 3305-3315 doi:10.1103/PhysRevC.58.3305

[30] P. K. Kakanis and G. S. Anagnostatos, "Persisting $\alpha$-Planar Structure in ${ }^{20} \mathrm{Ne}$," Physical Review C, Vol. 54, No. 6, 1996, pp. 2996-3013. doi:10.1103/PhysRevC.54.2996
[31] G. S. Anagnostatos, "Classical Equations-of-Motion Model for High-Energy Heavy-Ion Collisions," Physical Review C, Vol. 39, No. 3, 1989, pp. 877-883. doi:10.1103/PhysRevC.39.877

[32] G. S. Anagnostatos and C. N. Panos, "Semiclassical Simulation of Finite Nuclei," Physical Review C, Vol. 42, No. 3, 1990, pp. 961-965. doi:10.1103/PhysRevC.42.961

[33] G. S. Anagnostatos, "Towards A Unification of Independent and Collective Models, $20^{\text {th }}$ Conference of the Hellenic Nuclear Physical Society, Athens, May 27-28, 2011 (PDF in: www.uoi.gr/HNPS). 